\title{
Anti-CD11a Ameliorates Disease in the Human Psoriatic Skin-SCID Mouse Transplant Model: Comparison of Antibody to CD11a with Cyclosporin A and Clobetasol Propionate
}

\author{
Mary Zeigler, Yiqing Chi, Daniel B. Tumas, Sarah Bodary, Haicheng Tang, and \\ James Varani
}

Department of Pathology (MZ, YC, HT, JV), University of Michigan, Ann Arbor, Michigan; and Departments of Pathology and Immunology (DBT, SB), Genentech, Inc., South San Francisco, California

SUMMARY: The present study assesses the applicability of human skin-SCID (severe combined immunodeficiency) mouse chimeras in testing antipsoriatic therapeutics. Three agents were examined: (1) a monoclonal antibody to the alpha subunit of leukocyte function associated antigen-1 integrin (CD11a); (2) Cyclosporin A; and (3) clobetasol propionate (Temovate), a potent topical corticosteroid used clinically in the treatment of psoriasis. Skin transplanted to SCID mice from normal human volunteers or from psoriatic lesional skin was allowed to heal for 3 to 5 weeks before application of test reagents. During this period, psoriatic skin, which was 3.8-fold thicker than the corresponding normal skin before transplantation, maintained its phenotype (ie, increased epidermal thickness, rete ridges with blunted ends, and intralesional presence of T lymphocytes). Transplanted normal human skin, however, underwent a hyperplastic response during this period, resulting in a 2.4-fold increase in epidermal thickness. After the healing period, animals transplanted with normal or psoriatic skin were treated for 14 days by daily intraperitoneal injection of either Cyclosporin A or a monoclonal antibody to human CD11a, or by topical application of clobetasol propionate. At the end of the treatment period, the mice were killed and the tissue evaluated morphometrically for changes in epidermal thickness and immunohistologically for the presence of T lymphocytes. Both Cyclosporin A and anti-CD11a reduced the epidermal thickness of transplanted psoriatic skin, whereas neither reagent significantly reduced the thickness of transplanted normal skin. T lymphocytes were detected in the skin from treated animals; there did not seem to be any reduction in the number of T lymphocytes. Clobetasol propionate reduced the epidermal thickness of both normal and psoriatic skin. These data indicate that, in this model, therapies directed against pathophysiologic mechanisms that contribute to psoriasis can be distinguished from treatments that block epidermal hyperplasia occurring as a consequence of xenografting. Our observations provide evidence for the activity of anti-CD11a in an animal model of human psoriasis. (Lab Invest 2001, 81:1253-1261).

$P$ soriasis is a common inflammatory skin disease characterized by excessive keratinocyte proliferation, leading to epidermal thickening and expansion of epidermal rete pegs into papillary dermal space. An immunologic basis, possibly triggered by staphylococcal or streptococcal superantigen, is suspected, but the event that initiates formation of the psoriatic plaque is unknown (Valdimarsson et al, 1995). Abnormal epithelial differentiation, extensive capillary formation in the papillary dermis, and accumulation of inflammatory leukocytes including $\mathrm{T}$ lymphocytes, NK lymphocytes, and granulocytes are also consistent features (Fry, 1988; Krueger et al, 1984; Malhotra et al,

Received May 31, 2001

This study was supported in part by Grant CA 60958 from the United States Public Health Service.

Address correspondence to: Dr. James Varani, Department of Pathology, The University of Michigan, 1301 Catherine Road/Box 0602, Ann Arbor, MI 48109.E-mail:varani@umich.edu

Address reprint requests to: Dr. Sarah Bodary, Department of Immunology, MS34, Genentech, Inc., 1 DNA Way, South San Francisco, CA 940804990.E-mail:scb@gene.com
1989; Mils et al, 1994). In vitro studies show that lymphocytes interact with keratinocytes through leukocyte function-associated antigen-1 (LFA-1)-intercellular adhesion molecule-1 (ICAM-1) (Dustin et al, 1988). The interaction between keratinocytes, local antigen-presenting cells, and $\mathrm{T}$ lymphocytes likely initiates and maintains psoriatic lesions via the induction of cytokines, chemokines, and growth factors such as transforming growth factor- $\alpha$ (Austin et al, 1999; Elder et al, 1989; Goebeler et al, 1998; Nickoloff, 1991). Locally derived growth factors also contribute to epidermal hyperplasia in conditions other than psoriasis, for example, in wound healing (Stenn and Malhotra, 1992) and cancer (Gottlieb et al, 1988).

An impediment to the study of psoriasis is a lack of experimental models. No animal disease completely mimics psoriasis (Carroll et al, 1995; Sundberg et al, 1990). Keratinocytes and fibroblasts from psoriatic skin were isolated and studied in monolayer culture (Nickoloff et al 1989; Pellegrini et al, 1992; Priestley, 1987), and psoriatic lesional skin was studied in organ culture (Caron, 1968; Kondo, 1986; Kondo et al, 1992; Mils et al, 1994; Varani et al, 1998). Although some of 
the phenotypic characteristics of psoriatic lesional skin are maintained under in vitro culture conditions, other characteristics are lost over time (Kondo, 1986; Mils et al, 1994; Varani et al, 1998).

Transplantation of human skin onto immunocompromised mice (either congenitally athymic [nude] mice or severe combined immunodeficiency [SCID] mice) provides another approach to the study of psoriasis (Baker et al, 1992; Gilhar et al, 1997; Krueger, 1975; Krueger et al, 1981; Nickoloff et al, 1995; Nickoloff and Wrone-Smith, 1999; Wrone-Smith and Nickoloff, 1996). Using this approach, at least some of the phenotypic features of the disease (ie, epidermal thickening, extensive rete peg formation, and presence of inflammatory cells) are maintained for an extended period in the transplanted skin (Nickoloff et al, 1995). Although other characteristics of the psoriatic phenotype are lost over time, their loss seems to be retarded by co-injection of immune cells from psoriatic patients (Gilhar et al, 1997). Recent studies suggest that co-injection of circulating CD4positive lymphocytes from psoriatic patients not only preserves psoriatic features in lesional skin, but also induces expression of the psoriatic phenotype in nonlesional skin (Nickoloff and Wrone-Smith, 1999; Wrone-Smith and Nickoloff, 1996). Although these studies demonstrate the value of the human skinSCID mouse transplant model for investigation of the pathophysiology of psoriasis, it is not obvious from the published work how useful this approach may be for evaluating therapies intended to restore lesional tissue to a normal phenotype. To address this issue, we evaluated the abilities of three agents with known or anticipated antipsoriatic activity to modulate the phenotype of human psoriatic skin transplanted onto SCID mice. The agents evaluated were: Cyclosporin A (Wong et al, 1993); a monoclonal antibody to human CD11a (MHM-24, Genentech, Inc., South San Francisco, California) (Hildreth and August, 1985); and clobetasol proprionate, a potent topical steroid used clinically in the treatment of psoriasis and other dermatoses (Agarwal and Berth-Jones, 2000; Lebwohl et al, 2001). In parallel, the same agents were examined for effects on normal human skin transplanted onto SCID mice.

\section{Results}

Changes in Thickness of Normal and Psoriatic Skin after Transplantation onto SCID Mice: Effects of Cyclosporin A and Anti-CD11a

Figure 1 summarizes the epidermal thickness measurements, based on normal skin transplants from 5 volunteers and psoriatic lesional skin transplants from 10 volunteers. The data indicate that the normal skin was thinner than the psoriatic skin before transplantation. Seven weeks after transplantation, the thickness of the psoriatic skin was virtually unchanged (1.06-fold increase) from pretransplantation, whereas there was a dramatic increase in thickness of the transplanted normal skin (2.4-fold). With psoriatic skin, both Cyclosporin

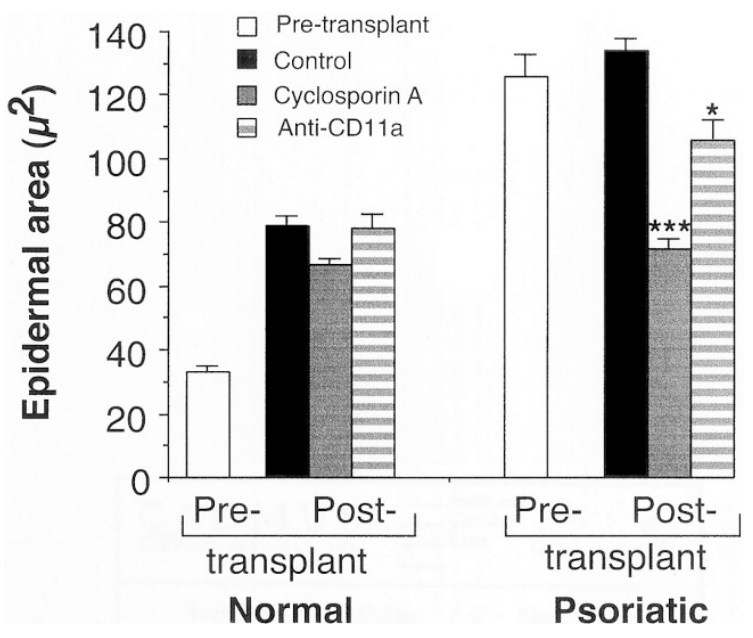

Figure 1.

Epidermal thickness of normal and psoriatic lesional skin immediately after biopsy and 7 weeks after transplantation onto severe combined immunodeficiency (SCID) mice. Animals were treated with PBS (control group), Cyclosporin A, or anti-CD11a for the last 14 days. Values shown represent the mean area of the epidermis (determined morphometrically) \pm standard error. Statistical significance of the differences among the various groups was determined by ANOVA, followed by paired-group comparisons. * Indicates difference from the post-transplantation control value at the $p<0.05$ level; *** indicates difference from the post-transplantation control value at the $p$ $<0.001$ level.

A and anti-CD11a treatments reduced the average epidermal thickness, compared with either psoriatic skin before transplantation or transplanted psoriatic skin treated with PBS. In contrast to these results with psoriatic skin, there was only a slight overall reduction in epidermal thickness of the normal skin treated with Cyclosporin A relative to the PBS-treated control, and no reduction with anti-CD11a.

Figure 2 shows typical histologic features of normal and psoriatic skin before transplantation and at the end of the treatment period. Differences in epidermal thickness of the normal and psoriatic skin before transplantation are apparent from the histology (Fig. 2, $A$ and E). The increase in epidermal thickness of the normal skin 7 weeks after transplantation onto the mouse is also apparent (Fig. 2, A and B). Histologically, 7-weeks after transplantation, normal skin had many of the features of psoriatic skin including expansion of epidermal rete pegs into papillary dermal space and an overall increase in epidermal thickness. In contrast to transplanted psoriatic skin, in which residual inflammatory cells (intra-epidermal neutrophils) were still visible after 7 weeks, there was no detectable inflammatory response associated with the hyperplastic normal skin. The lack of effect of the two agents on the histologic features of normal skin can be seen in Figure 2, C and D, whereas the reduction in epidermal thickness of the psoriatic skin after treatment with these two agents is evident in Figure 2, G and $\mathrm{H}$.

In addition to evaluating hematoxylin and eosinstained tissue sections, psoriatic skin grafts were also evaluated for CD3-positive human T lymphocytes before transplantation and after engraftment and treatment. CD3-positive cells were apparent in pre-transplantation 

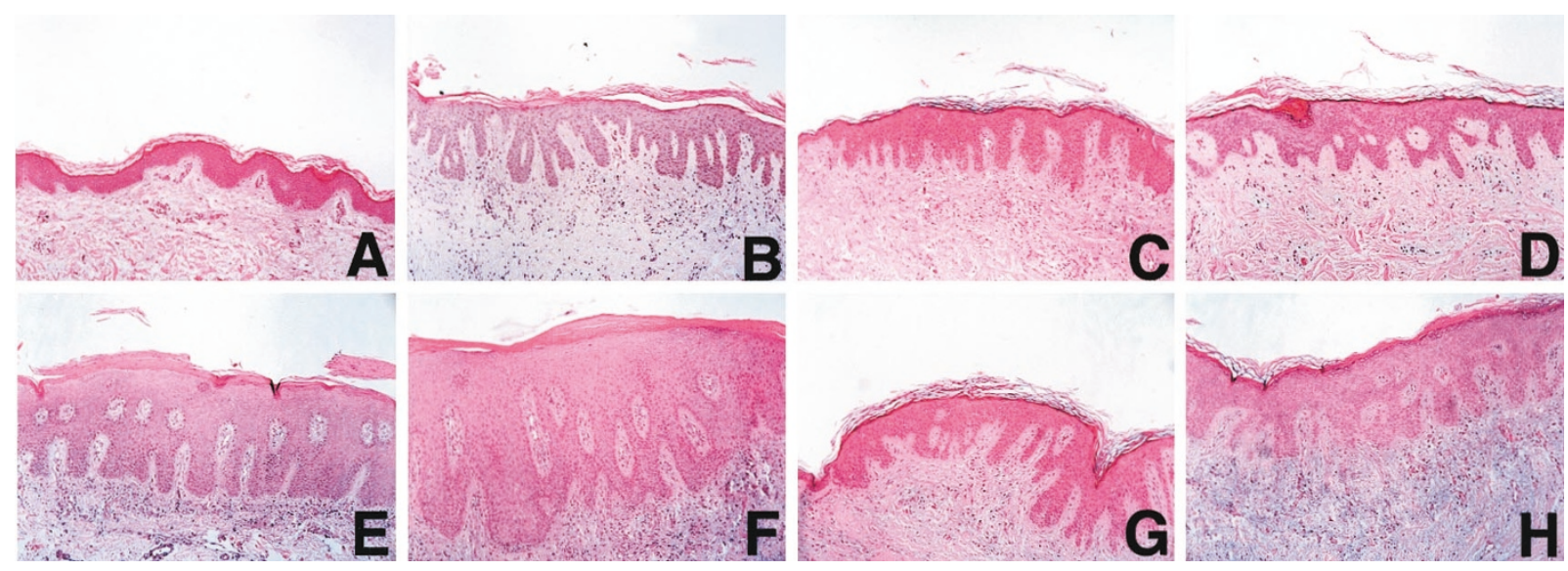

Figure 2.

Histologic appearance of normal skin and psoriatic lesional skin immediately after biopsy and 7 weeks after transplantation onto SCID mice. Animals were treated with PBS, Cyclosporin A, or anti-CD11a for the last 14 days. A, normal skin before transplantation; B, transplanted normal skin treated with PBS; C, transplanted normal skin treated with Cyclosporin A; D, transplanted normal skin treated with anti-CD11a; E, psoriatic skin before transplantation; F, transplanted psoriatic skin treated with PBS; G, transplanted psoriatic skin treated with Cyclosporin A; H, transplanted psoriatic skin treated with anti-CD11a. All sections were stained with hematoxylin and eosin; original magnification, $\times 160$.

psoriatic tissue, and in all psoriatic skin grafts after transplantation and treatment, CD3-positive T lymphocytes were maintained in the dermis with infiltration into the epidermis (Fig. 3).

\section{Effects of Clobetasol Propionate on Thickness of Normal and Psoriatic Skin after Transplantation onto SCID Mice}

Psoriatic skin from three additional donors and normal skin from two donors was transplanted onto SCID mice and treated topically with clobetasol propionate (once daily for 14 days) after allowing the skin to heal. At the end of the treatment period, tissue from treated and control animals was evaluated for epidermal thickness, as described above. Topical treatment with this potent synthetic steroid dramatically reduced epidermal thickness of both the normal and psoriatic skin (Fig. 4). Thinning was evident in the histologic sections of both tissues as was the reduction in rete ridges (Fig. $5)$.

\section{Discussion}

The human skin-SCID mouse transplant model provides a means for investigating the pathophysiology of psoriasis (Gilhar et al, 1997; Nickoloff and WroneSmith, 1999; Wrone-Smith and Nickoloff, 1996). Based on the findings presented in this report, we suggest that the human skin-SCID mouse transplant model may also be of use in the assessment of potential antipsoriatic therapies. Here we show that intraperitoneal treatment of SCID mice with two different agents, Cyclosporin A and a monoclonal antibody to human CD11a, caused a significant reduction in the thickness of transplanted human psoriatic plaque skin. At the same time, neither treatment significantly reduced the hyperproliferative response that occurred when normal human skin was transplanted onto SCID mice. Because $T$ cells are a major target of both Cyclosporin A (Jegasothy et al, 1992) and anti-CD11a
(Nakahura et al, 1993), the data presented here are consistent with the concept that therapies directed against $T$ cells can be effectively evaluated by this approach.

Although treatment with Cyclosporin $\mathrm{A}$ and antiCD11a both resulted in a statistically significant reduction in the thickness of the psoriatic epidermis, Cyclosporin A was quantitatively more effective. Although the reason for this discrepancy is unknown, one difference is that Cyclosporin A has broad affects on T cell function, including modulation of pro-inflammatory cytokine production (Wong et al, 1993), whereas a therapy directed against CD11a would presumably affect only the cell-cell interactions dependent on this receptor. The spectrum of cells targeted by the two agents is also different. In addition to its anti-T cell activity, Cyclosporin $\mathrm{A}$ has a number of other effects on a broad range of cell types that could foster antipsoriatic activity. Cyclosporin A inhibits keratinocyte growth in vitro (Karashima et al, 1996; Taniguchi et al, 1995), although its ability to do so in vivo is less clear (Bennett, 1997). In parallel with growth inhibition, Cyclosporin A reduces the production of pro-inflammatory effector molecules by keratinocytes (Iversen et al, 1996; Kaplan et al, 1995; Won et al, 1994). Fibroblasts, endothelial cells, and antigen-presenting cells may also be important targets of Cyclosporin $A$ in psoriasis (Fukuoka et al, 1998; Murray et al, 1998; Wong et al, 1993). Effects on any of these populations could contribute to the antiproliferative and anti-inflammatory effects of Cyclosporin A. In contrast, a monoclonal antibody directed against CD11a would be expected to act solely on CD11a-expressing cells. CD11a/CD18 (LFA-1) is expressed on all leukocytes and its primary function is to mediate the interaction of T cells with other cell types, including B cells, dendritic cells, endothelial cells, and epithelial cells. Just as the capacity of Cyclosporin A to affect multiple cell types in addition to T cells may explain its greater antipsoriatic activity than antiCD11a, this same property may also underlie the side 

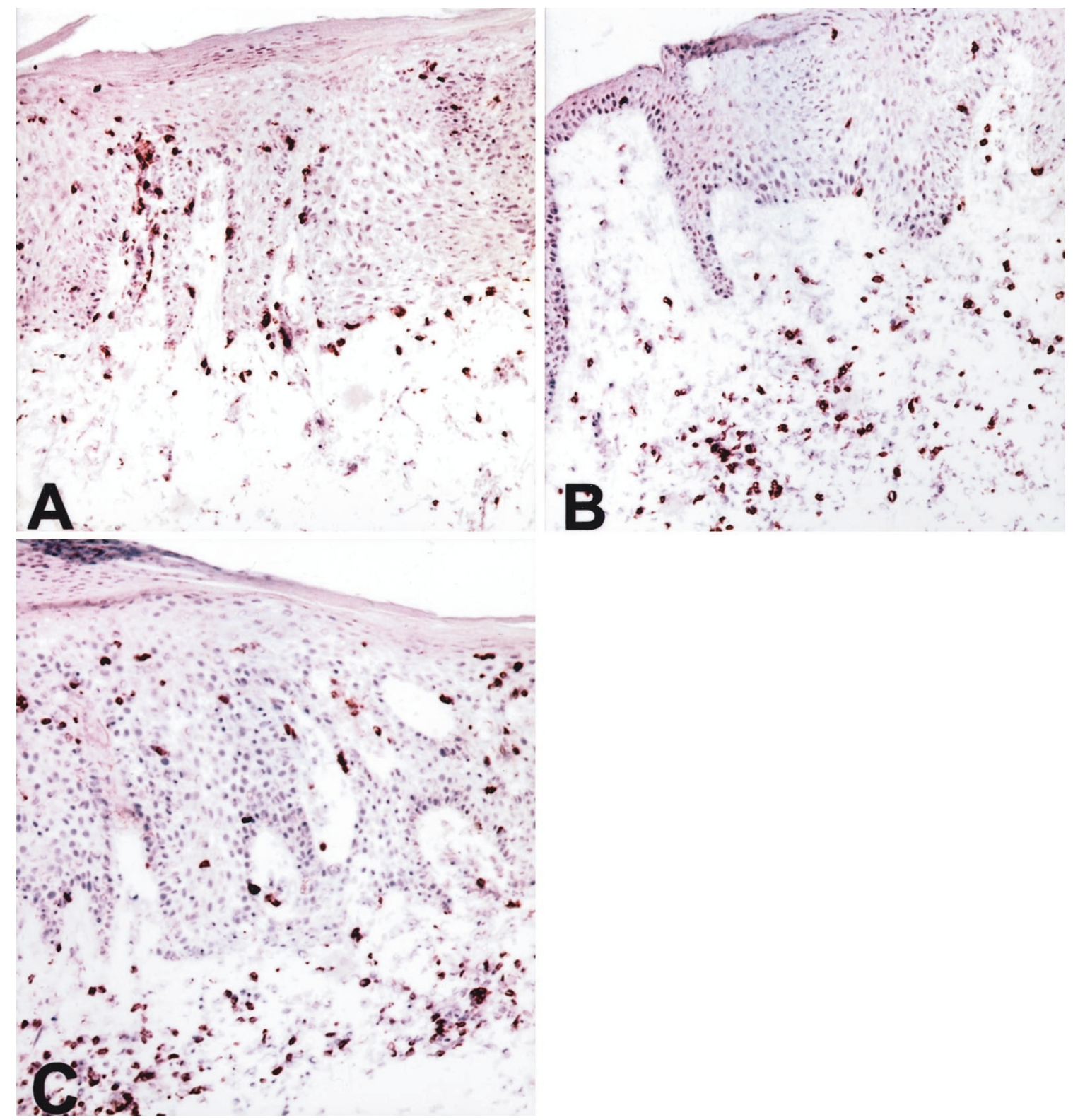

Figure 3.

Immunochemical analysis of T lymphocytes in psoriatic skin grafts after treatment. Immunochemistry was performed on frozen sections of psoriatic lesional skin after treatment with an monoclonal antibody to human CD3. For each treatment group, saline control (A), monoclonal antibody to CD11a, MHM24 (B), and Cyclosporin A $(C)$, there was a similar mild infiltration of $T$ lymphocytes into the graft at the end of the treatment period.

effects associated with Cyclosporin A. The use of Cylcosporin A in psoriasis is limited by its potential sclerosing effects in the kidney (Bennett, 1997). It is anticipated that a therapeutic such as anti-CD11a, a leukocytespecific agent, would not produce similar changes in glomerular structure.

Alternatively, the human skin-SCID mouse transplant model may systematically underestimate the effectiveness of therapies directed specifically at $T$ lymphocytes. A therapy directed against CD11a would be expected to inhibit $T$ lymphocyte recruitment to lesional skin as well as cell-cell interactions within the lesion. Recent data from human clinical trials suggests that this may be the basis for the improvement in psoriasis after treatment with anti-CD11a (Gottlieb et al, 2000). Because the xenograft model relies on human lymphocytes already present in the tissue at the time of transplantation, antibody effectiveness in this model would seem to depend on its ability to interfere with the functions of cells that are already in the lesion. Indeed, we saw no change in the presence of T lymphocytes in the grafted skin after any of the treatments. This suggests that anti-CD11a treatment was not cytoxic to these cells, and is in agreement with the leukocytosis observed in human and murine studies with antibodies to CD11a (Connolly et al 1994; Gottlieb et al, 2000). It follows, therefore, that the functioning of $\mathrm{T}$ lymphocytes in the psoriatic lesion can be blunted without eliminating effector cells from the lesion. 


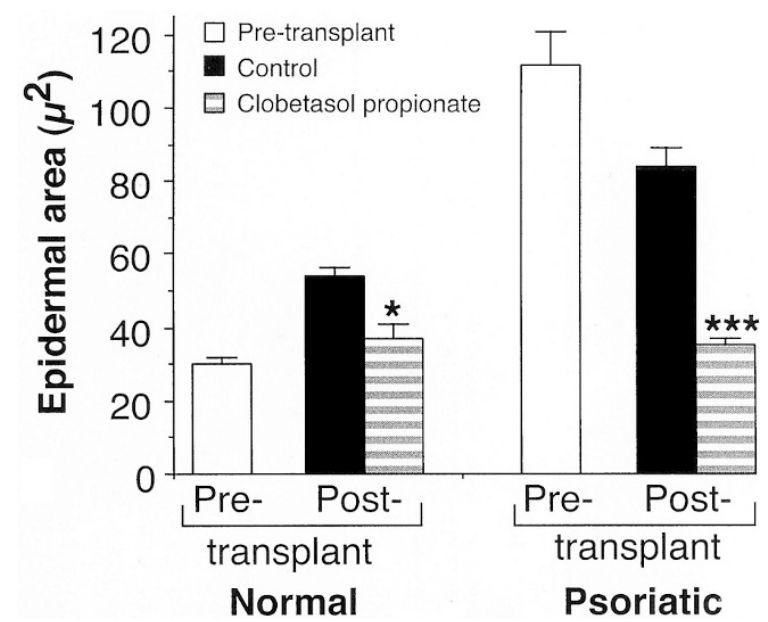

Figure 4.

Epidermal thickness of normal and psoriatic lesional skin immediately after biopsy and 7 weeks after transplantation onto SCID mice. Animals were treated topically with clobetasol propionate for the last 14 days. Values shown represent the mean area of the epidermis (determined morphometrically) \pm standard error. Statistical significance of the differences among the various groups was determined by ANOVA, followed by paired-group comparisons. * Indicates difference from the post-transplantation control value at the $p<0.05$ level; ${ }^{* \star}$ indicates difference from the post-transplantation control value at the $p<0.001$ level.

Finally, although the level of efficacy with antiCD11a was significant, increased efficacy might be seen with a longer duration of treatment, as suggested by results from a recent clinical trial in human patients with psoriasis. Clinical trial data indicated that although significant benefit, including a reduction in epidermal thickness, could be seen 4 weeks after a single dose of anti-CD11a, additional improvement was seen at 8-weeks, as indicated by the Psoriasis Area and Severity Index score (Gottlieb et al, 2000).

The fact that neither Cyclosporin A nor anti-CD11a significantly reduced the hyperproliferation that occurred in transplanted normal skin strongly argues that the mechanism driving the hyperproliferative response in psoriatic plaque skin is different from the mechanism underlying the proliferative response in normal skin after transplantation. The induction of normal skin proliferation after transplantation does not seem, therefore, to prevent the use of human skin-SCID mouse chimeras for the study of antipsoriatic agents, although it does complicate the issue. This same phenomena also makes it more difficult to interpret findings related to induction of a psoriatic phenotype in nonlesional skin (Gilhar et al, 1997; Nickoloff and Wrone-Smith, 1999; Wrone-Smith and Nickoloff, 1996).

Findings with clobetasol propionate support this view. Treatment with this topical steroid dramatically reduced the epidermal thickness of transplanted psoriatic skin but also inhibited the hyperproliferative response in normal skin. Inhibition of cell growth and induction of skin atrophy is a well known consequence of topical steroid use (Haapasaari et al, 1996). Taken together with the Cyclosporin A and anti-CD11a findings, these data suggest that despite the hyperplastic changes occurring in transplanted normal skin, the psoriasis-specific component of the hyperproliferative response is preserved in this transplantation model. The ability of Cyclosporin A to reduce the thickness of psoriatic epidermis after transplantation to levels seen with normal skin might indicate an almost complete suppression of psoriasis-specific events.

The mechanism underlying the hyperplastic response in normal skin after transplantation is unknown at present. Some degree of epidermal hyperplasia is often seen as part of the wound-healing response in the skin (Stenn and Malhotra, 1992). Perhaps one or more growth factors present in the healing murine skin is responsible for triggering proliferation of epidermal keratinocytes in the transplanted human tissue. Consistent with this theory, we previously demonstrated that the same skin that becomes hyperplastic after transplantation onto SCID mice remains histologically normal when placed in organ culture in the absence of exogenous growth factors. Hyperplasia can be induced in organ-cultured skin by treatment with exogenous growth factors (Varani et al 1994, 1998).

Although these data indicate the applicability of the human psoriatic skin-SCID mouse transplantation model for evaluating potential antipsoriatic therapies, how useful the approach ultimately turns out to be will depend on a number of factors including ease of use and variability. Tissue availability is the major consideration for ease of use. Although psoriasis is common enough that an adequate number of volunteers can be identified, less tissue required from each individual means that more donors will be available and more experiments can be performed with the tissue. Reducing the size of the transplanted tissue piece is one potential way to reduce tissue limitations. Although our studies used keratome strips, a recent study describes successful transplantation of 8-mmdiameter human skin punch biopsies onto immunocompromised mice (White et al, 1999). Recently, we successfully transplanted 6-mm-diameter punch biopsies under the same conditions (M Zeigler and $\mathrm{J}$ Varani, unpublished observation). However, tissue size is ultimately limited by the ability to surgically handle the tissue piece and by the fact that pieces that are too small may be overgrown by the adjacent mouse skin (Boehncke et al, 1994). Another way to conserve tissue is to transplant each mouse with one rather than two tissue pieces. We used bilateral transplants in this study to ensure that if one tissue piece failed to engraft, the whole mouse would not be lost. However, of the 72 animals bilaterally transplanted, only three tissue grafts failed. In future experiments, animals could be unilaterally transplanted.

Another limitation imposed by tissue availability is the number of analytic assessments that can be made with each specimen. We used most of each transplanted tissue piece for morphometric analysis. A small piece was removed before fixation, placed in OCT, and frozen in liquid nitrogen. From the OCTfrozen tissue, enough sections were cut to evaluate $T$ lymphocyte marker expression. However, there was not sufficient OCT-frozen tissue to assess additional 

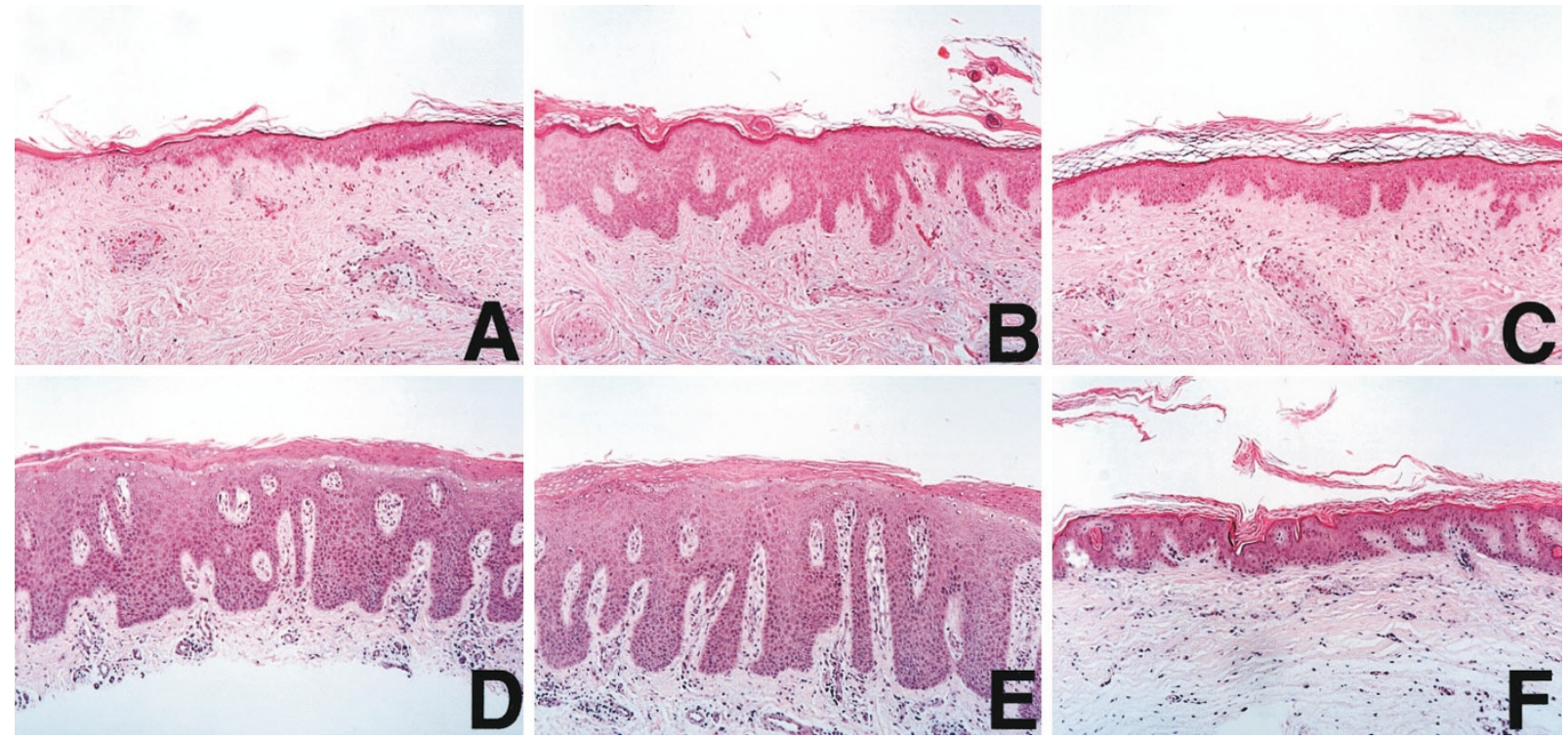

Figure 5.

Histologic appearance of normal skin and psoriatic lesional skin immediately after biopsy and 7 weeks after transplantation onto SCID mice. Animals were treated topically with clobetasol propionate for the last 14 days. A, normal skin before transplantation; B, transplanted normal skin treated with PBS; C, transplanted normal skin treated with clobetasol propionate; D, psoriatic skin before transplantation; E, transplanted psoriatic skin treated with PBS; F, transplanted psoriatic skin treated with clobetasol propionate. All sections were stained with hematoxylin and eosin; original magnification, $\times 160$.

antigens. Increasing the size of the frozen tissue piece would have left less tissue for the morphometric analysis, which could have compromised the major analytic endpoint. Ultimately, one needs to carefully consider what analytic measurements are to be made with each tissue piece.

Finally, variability is a concern. Analysis of the complete data set revealed that epidermal thickness measurements varied from patient to patient, from multiple mice with transplanted skin from a single patient and from transplant to transplant (between tissue pieces from two patients transplanted onto one mouse). Another major source of variability was from area to area within each tissue piece. The implication is that the epidermal thickness of psoriatic lesional plaque skin is highly not uniform. Therefore, preparing several histologic sections from each transplant and examining multiple segments across the entire histologic section is as important as having multiple tissue donors and transplanting multiple mice with skin from each donor.

In summary, this report describes the use of human psoriatic skin-SCID mouse chimeras for evaluation of potential antipsoriatic agents. We conclude that although complicated by hyperproliferative changes that occur in normal skin simply as a consequence of transplantation onto the mouse, this transplantation model can be used to evaluate agents for antipsoriatic efficacy. Our data also suggest that therapies directed against psoriasis-specific pathophysiologic mechanisms can be distinguished from treatments that nonspecifically block epidermal hyperplasia. Finally, in conjunction with recently reported early clinical results attained with anti-CD11 therapy (Gottlieb et al, 2000), the efficacy demonstrated for the monoclonal antibody to CD11a tested in this study provides a sound rationale for the continued development of this novel approach to the treatment of psoriasis.

\section{Materials and Methods}

\section{Normal Skin and Psoriatic Lesional Skin}

One or two full-thickness keratome strips $(7.5 \times 2.5$ $\mathrm{cm}$ ) consisting of the entire epidermis and several millimeters of dermis of normal human skin were obtained from the hips of seven healthy adult volunteers using an Aesculap Dermatome (Aesculap, Tuttlingen, Germany). None of the volunteers had a history of psoriasis and none were on systemic or topical medication for any condition at the time of tissue donation. Keratome strips of psoriatic lesional skin $(7.5 \times 2.5 \mathrm{~cm})$ were also obtained (before treatment) from 13 volunteers during flareups of the disease. Individuals were recruited in accordance with the guidelines of the University of Michigan Institutional Review Board and biopsied after receiving informed consent.

\section{Reagents}

Cyclosporin A was obtained from SANDOZ Pharmaceuticals Corporation (East Hanover, New Jersey). The murine antibody to human CD11a used was MHM-24 (Genentech, Inc., South San Francisco, California) (Hildreth and August, 1985). Clobetasol propionate (Temovate) was obtained from Glaxo Wellcome Inc. (Research Triangle Park, North Carolina), formulated by the manufacturer as an emollient cream containing $0.05 \%$ reagent. 


\section{Tissue Transplantation and Treatment Protocols}

SCID mice (CB-17 strain; Taconic Farms Inc., Germantown, New York) were used as tissue recipients. One to three keratomed tissue samples were obtained from each normal or psoriatic volunteer and cut into 1 $\times 1 \mathrm{~cm}$ sections. Two to four mice were transplanted bilaterally with each human skin sample, depending on tissue availability. After mice were anesthetized by intraperitoneal injection of sodium pentobarbital (Butler Company, Columbus, Ohio) at a dose of $1.8 \mathrm{mg}$ in $0.2 \mathrm{ml}$ per $25 \mathrm{gm}$ body weight, the dorsal region of each mouse was shaved bilaterally. Mouse skin was surgically removed to size, and replaced with the human tissue. The transplanted tissue was secured to the back of the mouse with absorbable sutures (4-0 Dexon "S"; Davis-Geck, Manati, Puerto Rico). The transplants were bandaged with Xeroform petrolatum dressing (Kenadall Company, Mansfield, Massachusetts) for 5 days. The animals were maintained in a pathogen-free environment throughout the preparation and treatment phases. Treatment was initiated 3 to 5 weeks after transplantation. Transplanted animals were divided into treatment groups (vehicle plus test reagents) or a nontreatment group (vehicle alone). Cyclosporin A was delivered by intraperitoneal injection at $20 \mathrm{mg} / \mathrm{kg}$ body weight in $100 \mu \mathrm{L}$ of PBS. The monoclonal antibody to CD11a was also delivered intraperitoneally in $100 \mu \mathrm{L}$ of PBS $(6 \mathrm{mg} / \mathrm{kg}$ of body weight). The antibody concentration was chosen based on comparable activity to Cyclosporin $A$ in the murine heart transplant model (Nakahura et al, 1993). The control mice were treated with PBS alone. Treatment was continued daily for 14 days. Clobetasol propionate emollient cream was applied topically to the transplanted tissue daily for 14 days. All procedures involving animals were approved by the University Committee on Use and Care of Animals.

\section{Quantitative Evaluation of Epidermal Thickness}

After the treatment phase, mice were killed and the transplanted human tissue surgically removed and fixed in $10 \%$ formalin. After paraffin embedding, one to three 5 - $\mu \mathrm{m}$-thick sections were cut from each tissue piece, mounted onto microscope slides, and stained with hematoxylin and eosin. The epidermal area was measured as a function of changes in epidermal thickness per unit length using $\mathrm{NIH}$ Image software (National Institutes of Health, Bethesda, Maryland). Specifically, tissue sections were visualized by light microscopy at $\times 10$ magnification. At this level of magnification, the entire epidermal area of each tissue section was "captured" in equal segments (three to four segments across a typical tissue section), and the area of each segment was quantified using the NIH Image analysis program. Multiple areas from bilateral transplants on two to four mice per treatment group for each donor were quantified in this way, to provide 100 or more measurements. The mean epidermal area was determined from these values. Before transplantation, a small piece of tissue from each donor was fixed in $10 \%$ buffered formalin and used for zerotime assessment of epidermal thickness.

\section{Histology and Immunohistochemical Assessment}

To demonstrate that psoriatic skin grafts had histologic characteristics of psoriasis including epidermal hyperplasia, increased rete peg formation, and dermal and/or intra-epidermal infiltration with lymphocytes and neutrophils, 5-mm-thick sections were obtained from each tissue piece before transplantation, stained with hematoxylin and eosin, and evaluated microscopically.

To demonstrate the presence of infiltrating $\mathrm{T}$ lymphocytes within the grafts and to verify the maintenance of this population of cells after engraftment, anti-CD3 immunohistochemistry was performed. For each psoriatic skin biopsy, frozen sections were obtained before transplantation onto the SCID mice, and after transplantation and treatment. Using a Vectastain Elite ABC Kit (Vector, Burlingame, California), a biotin-labeled monoclonal antibody to human CD3 (1 $\mu \mathrm{g} / \mathrm{ml}$; mAb A0452; DAKO, Carpinteria, California) was applied for 30 minutes to frozen tissue sections after fixation with acetone and ethanol, quenching of endogenous peroxidase activity, blockade for endogenous biotin (Vector Avidin/Biotin Blocking Kit; Vector), and blockade of immunoglobulin-binding sites with $10 \%$ normal goat serum.

\section{Statistical Analysis}

Measurements of epidermal thickness for each group of tissues (pre-transplantation, post-transplantation control, and post-transplantation treated) were analyzed by ANOVA and comparisons between paired groups. The analysis accounted for the correlation between pre-treatment values and post-treatment values for each individual tissue donor, using a mixed model approach (Henderson, 1990; Searle et al, 1992).

\section{Acknowledgements}

The authors thank James Reimann for statistical analysis, Robin Kunkel and Lisa Riggs for morphometric analysis, and Robin Taylor, Patti Tobin, and Barbara Wright for histologic processing and immunohistochemistry.

\section{References}

Agarwal S and Berth-Jones J (2000). A contact dermatitis reaction to clobetasol propionate cream associated with resolution of recalcitrant, generalized granuloma annulare. J Dermatol Treatment 11:279-282.

Austin LM, Ozawa M, Kikuchi T, Walters IB, and Krueger JG (1999). The majority of epidermal T cells in psoriasis vulgaris lesions can produce type 1 cytokines, interferon- $\gamma$, interelukin-2, and tumor necrosis factor- $\alpha$, defining TC1 (cytotoxic T lymphocyte) and TH1 effector populations: A type 1 differentiation bias also measured in circulating blood T cells in psoriasis patients. J Invest Dermatol 113:101-108.

Baker BS, Brent L, Valdimarsson H, Powles AV, al-Imara L, Walker M, and Fry L (1992). Is epidermal proliferation in psoriatic skin grafts on nude mice driven by T-cell derived cytokines? Br J Dermatol 126:105-110. 
Bennett WM (1997). Cyclosporin nephrotoxicity: Implications for dermatology. Int J Dermatol 36(Suppl 1):11-14.

Boehncke WH, Sterry W, Hainzl A, Scheffold W, and Kaufmann R (1994). Psoriasiform architecture of murine epidermis overlaying human psoriatic dermis transplanted to SCID mice. Arch Dermatol Res 286:325-330.

Caron CA (1968). Organ culture of normal and psoriatic skin. Arch Dermatol 97:575-586.

Carroll JM, Romero MR, and Watt FM (1995). Suprabasal integrin expression in the epidermis of transgenic mice results in developmental defects and a phenotype resembling psoriasis. Cell 83:957-968.

Connolly KA, Kitchens AK, Chan B, Jardieu P, and Wofsy D (1994). Treatment of murine lupus with monoclonal antibodies to LFA-1. Clin Immunol Immunopath 72:198-203.

Dustin ML, Singer KH, Tuck DT, and Springer TA (1988). Adhesion of $\mathrm{T}$ lymphoblasts to epidermal keratinocytes is regulated by interferon- $\gamma$ and is mediated by intercellular adhesion molecule-1. J Exp Med 167:1323-1340.

Elder JT, Fisher GJ, Lindquist PB, Bennett GJ, Pittelkow MR, Coffey RJ, Ellingsworth L, Derynck R, and Voorhees JJ (1989). Overexpression of transforming growth factor- $\alpha$ in psoriatic epidermis. Science 243:811-814.

Fry L (1988). Psoriasis. Br J Dermatol 119:445-461.

Fukuoka M, Ogino Y, Sato H, Ohta T, and Komoriya K (1998). Regulation of RANTES and IL-8 production in normal human dermal fibroblasts by active vitamin D3. $\mathrm{Br} \mathrm{J}$ Pharmacol 124:1433-1438.

Gilhar A, David M, Ullmann Y, Berkutski T, and Kalish RS (1997). T-lymphocyte dependence of psoriatic pathology in human psoriatic skin grafted to SCID mice. J Invest Dermatol 109:283-288.

Goebeler M, Toksoy A, Spandau U, Engelhardt E, Broker EB, and Gillitzer R (1998). The C-X-C chemokine Mig is highly expressed in the papillae of psoriatic lesions. J Pathol 184:89-95.

Gottlieb AB, Chang CK, Posnett DN, Fanelli B, and Tam JP (1988). Detection of transforming growth factor- $\alpha$ in normal, malignant, and hyperproliferative human keratinocytes. J Exp Med 167:670-675.

Gottlieb A, Kreuger JG, Bright R, Ling M, and Garavoy M (2000). Effects of administration of a single dose of a humanized monoclonal antibody to CD11a on the immunobiology and clinical activity of psoriasis. J Am Acad Dermatol 42: $428-435$.

Haapasaari K-M, Risteli J, and Oikarinen A (1996). Recovery of human skin collagen synthesis after short-term topical corticosteroid treatment and comparison between young and old subjects. Br J Dermatol 135:65-69.

Henderson CR (1990). Statistical methods in animal improvement: Historical overview. In: Advances in statistical methods for genetic improvement of livestock. New York: Springer-Verlag, 1-14.

Hildreth JEK and August JT (1985). The human lymphocyte function-associated (HLFA) antigen and a related macrophage differentiation antigen (Hmac-1): Functional effects of subunit-specific monoclonal antibodies. J Immunol 134: 3272-3280.
Iversen L, Syendsen M, and Kragballe K (1996). Cyclosporin A down-regulates LTA4 hydrolase level in human keratinocyte cultures. Acta Derm Venereol 76:424-428.

Jegasothy BV, Ackerman CD, Todo S, Fung JJ, Abu-Elmagd K, and StarzI TE (1992). Tacrolimus (FK506): A new therapeutic agent for recalcitrant psoriasis. Arch Dermatol 128:781785.

Kaplan A, Matsue H, Shibaki A, Kawashima T, Kobayashi H, and Ohkawara A (1995). The effects of cyclosporin A and FK506 on proliferation and IL-8 production of cultured human keratinocytes. J Dermatol Sci 10:130-138.

Karashima T, Hachisuka H, and Sasai Y (1996). FK506 and cyclosporin A inhibit growth factor-stimulated keratinocyte proliferation by blocking cells in the G0/G1 phases of the cell cycle. J Dermatol Sci 12:246-254.

Kondo S (1986). Maintenance of epidermal structure of psoriatic skin in organ culture. J Dermatol 13:242-249.

Kondo S, Hozumi Y, Maejima H, and Aso K (1992). Organ culture of psoriatic skin: Effect of TGF- $\alpha$ and TGF- $\beta$ on epidermal structure in vitro. Arch Dermatol Res 284:150-153.

Krueger GC (1975). Long-term maintenance of psoriatic human skin on congenitally athymic (nude) mice. J Invest Dermatol 64:307-312.

Krueger GC, Bergstresser PR, Lowe NJ, Voorhees JJ, and Weinstein GD (1984). Psoriasis. J Am Acad Dermatol 11:937947.

Krueger GC, Chambers DA, and Shelby J (1981). Involved and uninvolved skin from psoriatic subjects: Are they equally diseased? Assessment by skin transplantation to congenitally athymic (nude) mice. J Clin Invest 68:1548-1557.

Lebwohl M, Lombardi K, and Tan MH (2001). Duration of improvement in psoriasis after treatment with tazarotene plus clobetasol propionate: Comparison of maintenance treatments. Int J Dermatol 40:64-66.

Malhotra R, Stenn KS, Fernandez LA, and Braverman IM (1989). Angiogenic properties of normal and psoriatic skin associate with epidermis, not dermis. Lab Invest 61:162-165.

Mils V, Basset-Seguin N, Moles J-P, Tesniere A, Leigh I, and Guilhow J-J (1994). Comparative analysis of normal and psoriatic skin both in vivo and in vitro. Differentiation 58:7786.

Murray AG, Schechner JS, Epperson DE, Sultan P, McNiff JM, Hughes CC, Lorger MI, Askenase PW, and Pober JS (1998). Dermal microvascular injury in the human peripheral blood lymphocyte reconstituted-SCID mouse allograft model is $T$ cell mediated and inhibited by a combination of cyclosporin and rapamycin. Am J Pathol 153:627-638.

Nakahura EK, McCabe SM, Zheng B, Shorthouse RA, Scheiner TM, Blank G, Jardieu PM, and Morris RE (1993). Potent and effective prolongation by anti-LFA-1 monoclonal antibody monotherapy of non-primarily vascularized heart allograft survival in mice without $\mathrm{T}$ cell depletion. Transplantation 55:412-417.

Nickoloff BJ (1991). The cytokine network in psoriasis. Arch Dermatol 127:871-884

Nickoloff BJ, Kunkel SL, Burdick M, and Strieter RM (1995). Severe combined immunodeficiency mouse and human psoriatic skin chimeras. Validation of a new animal model. Am J Pathol 146:580-588. 
Nickoloff BJ, Mitra RS, Elder JT, Fisher GJ, and Voorhees JJ (1989). Decreased growth inhibition by recombinant gamma interferon is associated with increased production of transforming growth factor alpha in keratinocytes cultured from psoriatic lesions. Br J Dermatol 121:161-174.

Nickoloff BJ and Wrone-Smith (1999). Injection of prepsoriatic skin with CD4+ T cells induces psoriasis. Am J Pathol 155:145-158.

Pellegrini G, DeLuca M, Orecchia G, Balzac F, Cremona O, Savoia P, Cancedda R, and Marchisio PC (1992). Expression, topography and function of integrin receptors are severely altered in keratinocytes from involved and uninvolved psoriatic skin. J Clin Invest 89:1783-1795.

Priestley GC (1987). Proliferation and glycosaminoglycan secretion in fibroblasts from psoriatic skin: Differential response to retinoids. Br J Dermatol 117:575-583.

Searle SR, Casella G, and McCulloch CE (1992). Variance components. New York: John Wiley \& Sons, Inc.

Stenn KS and Malhotra R (1992). Epithelialization. In: Cohen IK, Diegelmann RF, and Lindblad WJ, editors. Wound healing: Biochemical and clinical aspects. Philadelphia: W. B. Saunders Company, 115-129.

Sundberg JP, Beamer WG, Shultz LD, and Dunstan RW (1990). Inherited mouse mutations as models of human adnexal, cornification, and papulosquamous dermatoses. J Invest Dermatol 95(Suppl):62s-63s.

Taniguchi S, Kono T, Ishii M, Otani S, and Hamada T (1995). Effects of cyclosporin and ultraviolet radiation on growth and ornithine decarboxylase activity in cultured epidermal keratinocytes. Br J Dermatol 132:703-709.
Valdimarsson H, Baker BS, Jonsdottir I, Powles A, and Fry L (1995). Psoriasis: A T-cell mediated autoimmune disease mediated by streptococcal superantigen? Immunol Today 16:145-149.

Varani J, Kang S, Stoll S, and Elder JT (1998). Human psoriatic skin in organ culture: Comparison with normal skin exposed to exogenous growth factors and effects of an antibody to the EGF receptor. Pathobiology 66:253-259.

Varani J, Perone P, Griffiths CEM, Inman D, Fligiel SEG, and Voorhees JJ (1994). All-trans retinoic acid stimulates events in organ-cultured skin that underlie repair. J Clin Invest 94:1747-1753.

White PJ, Fogarty RD, Liepe IJ, Delaney PM, Werther GA, and Wraight CJ (1999). Live confocal microscopy of oligonucleotide uptake by keratinocytes in human skin grafts on nude mice. J Invest Dermatol 112:887-892.

Won YH, Sauder DN, and McKenzie RC (1994). Cyclosporin A inhibits keratinocyte cytokine gene expression. Br J Dermatol 130:312-319.

Wong RL, Winslow CM, and Cooper KD (1993). The mechanisms of action of cyclosporin $A$ in the treatment of psoriasis. Immunol Today 14:69-74.

Wrone-Smith T and Nickoloff BJ (1996). Dermal injection of immunocytes induces psoriasis. J Clin Invest 98:1878-1887. 